\title{
Share your learning points: an opportunity for you to publish
}

The RCGP is planning to publish a book entitled The General Practice Book of Golden Rules and Reflective Learning. We all learn by experience, but how often is such learning lost because we have not put pen to paper? The purpose of the book is to harness and record valuable learning points in order to hand them down to the next generation. The publication will comprise reflective learning pieces derived from real-life experiences in general practice, followed by 'golden rules' encapsulating the learning points and illustrated with cartoons. It is hoped that it will have a significant role in teaching. Each golden rule or reflective learning piece could be the subject of a tutorial in itself, or the book could be used as an 'ice breaker' at the beginning of group work.
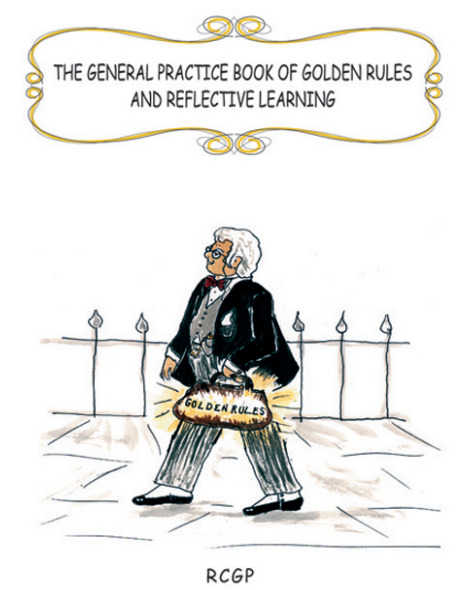

The College is seeking further submissions of reflective learning pieces (if possible with accompanying golden rules) for inclusion in the first edition. All contributing authors will be acknowledged in the preface, but in order not to discourage reflective learning pieces of a sensitive nature, authorship of submissions will not be individually identifiable.

Published reflective learning pieces will be displayed within a tasteful ornate frame on the page adjacent to the relevant golden rule and cartoon.

At the end of the book, pages will be provided for readers to record their own reflective learning pieces and golden rules, which they could then send into the College for inclusion in the next edition.

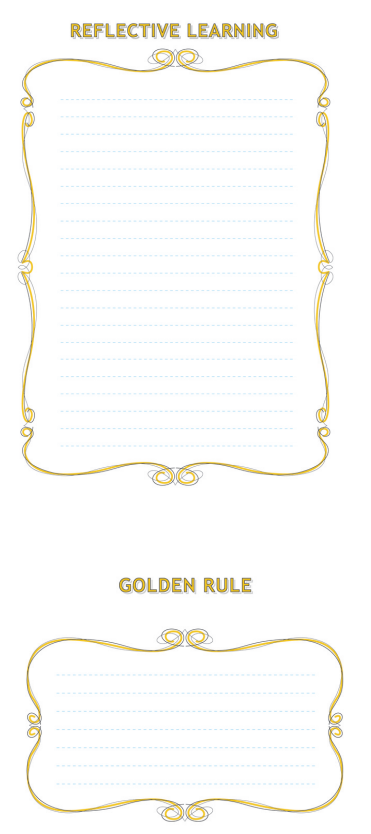

The RCGP will be pleased to consider submissions ranging from a single paragraph to 1000 words. Please feel free to describe a suitable cartoon if you so wish.

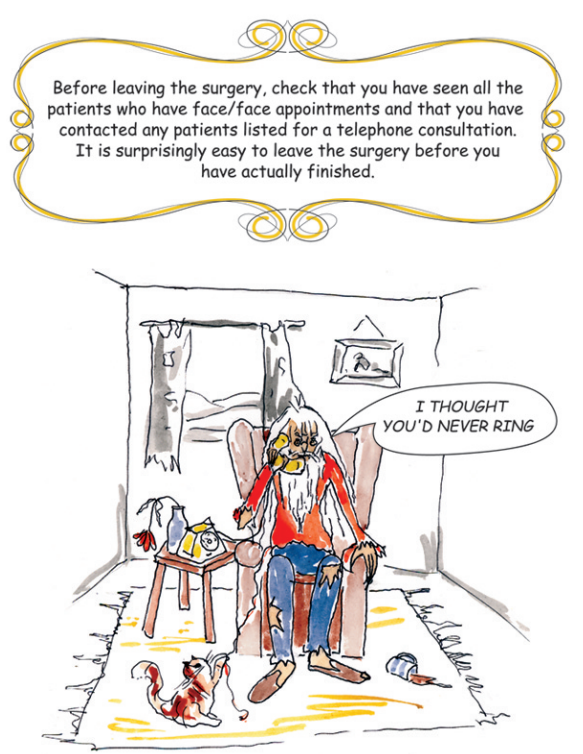

The following are examples of suitable reflective learning pieces, golden rules, and cartoon descriptions: A few illustrations are included to convey the general character of the book.

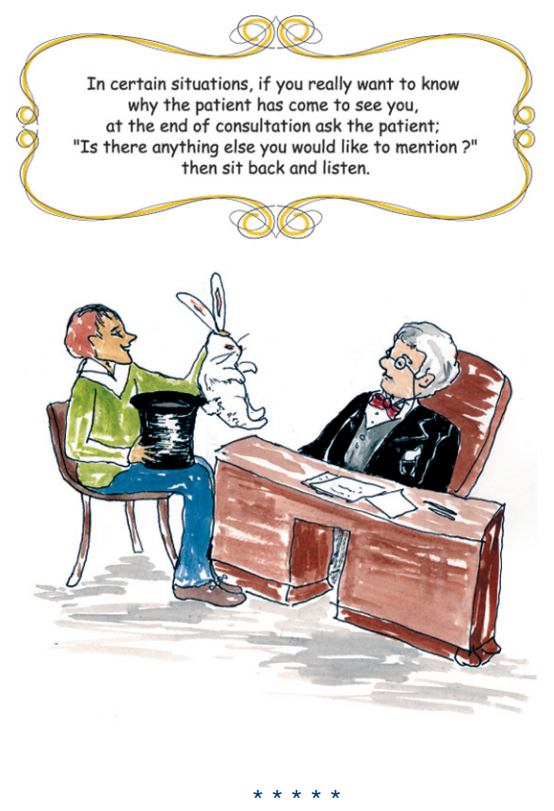

\section{I can't walk in a straight line}

A 66-year-old male patient, with a history of depression and high alcohol intake, who had been taking paroxetine for approximately 3 years, presented with symptoms of confusion, nausea, dizziness, euphoric episodes, and difficulty in coordinating movements. Examination revealed myoclonus and hypereflexia. I looked on an electronic drug database and made a diagnosis of the 'serotonin syndrome' occurring as a side effect of paroxetine therapy.

I referred the patient to hospital where routine haematological and biochemical investigations were found to be normal. He was observed overnight, advised to reduce the paroxetine slowly, and was prescribed a short course of chlordiazepoxide.

The patient, however, stopped the paroxetine abruptly, and after a short period his symptoms resolved. 


\section{They've moved the front door and the house is full of mice}

An 81-year-old female patient with Type 2 diabetes on insulin, polymyalgia rheumatica, hypertension, gastric ulcer, and hypercholesterolaemia was prescribed sibutramine for weight reduction. On day 4 she developed marked confusion, disorientation, hallucinations and agitation together with unsteadiness.

Her symptoms continued. After 39 days treatment with sibutramine, a diagnosis of the serotonin syndrome was made. The sibutramine was discontinued and her symptoms resolved within a few days.

The diagnosis of the serotonin syndrome is especially important in patients in this age group as it can so easily be confused with senile dementia. Patients could therefore be unnecessarily institutionalised if the true cause of their symptoms is not identified.

Golden Rule: Before initiating any investigation or treatment, ensure that the patient's symptoms are not due to a drug side effect.

Cartoon: Doctor sitting with patient. Patient glowing iridescent green. Bubble from patient saying 'But really Doctor, I don't think this medicine is quite agreeing with me'.

\section{It may run in the family}

A 25-year-old male patient presented with a left-sided foot drop following a fall downstairs. I recalled that his 22-year-old brother had presented the previous year with a left-sided brachial plexus injury sustained while moving a filing cabinet. I wondered if there was a familial condition that predisposed the patient to nerve damage following relatively minor trauma. I telephoned a neurologist colleague who confirmed that such a condition did exist, and was known as 'hereditary neuropathy with liability to pressure palsies'. He informed me that the condition is due to mutations in the peripheral myelin protein 22 gene on chromosome 17 which probably cause myelin to become unstable. I subsequently discussed the case with a clinical geneticist who informed me that the condition is inherited as an autosomal dominant trait, and that the family could be screened. Having obtained permission from both brothers, I offered screening to the family, who to date, have not taken up the offer.

Golden Rule: If two or more members of the same family present with a rare or unusual condition, explore the possibility of a familial/hereditary condition.

Cartoon: A nuclear family standing in line, all with purple frizzy hair.

\section{Discussing personal information}

Many years ago, a patient attended the surgery and told me she was having marital problems. I asked her to tell me about it. She replied that she would rather not. Ever since then, in such situations, I have used language such as 'Do you feel you would like to talk about it?'

Golden Rule: When the consultation involves discussing personal information that the patient may or may not want to tell you, metaphorically open the door, but don't drag them in. Use language such as, 'Do you feel you would like to talk about it?'

Cartoon: A Doctor holding the consultation room door open and dragging a protesting patient in.

Golden Rule: If rapport of a personal/sensitive nature is established, keep to the right side of the line: constructive information-seeking versus intrusive nosiness.

Cartoon: The statue of Justice holding the scales. In the left scale pan a bag labelled 'constructive information seeking' in the right scale pan, a bag labelled 'intrusive nosiness'. The left scale pan lower than the right scale pan.

$$
* * * * *
$$

You may write about any topic(s) that you wish, but here are a few suggestions.

- An unexpected benefit of a drug

- How a communication from a pharmacist prevented a prescribing error

- Something that made me feel good in everyday practice

- How a patient proffered a management option

- An extensively investigated symptom that turned out to be a side effect of the patient's medication

- Placating an angry patient

- How a patient who kept presenting with the same symptom was eventually diagnosed

- A prescribing error/safe prescribing

- A breakdown in communication

- Diagnosis

- Problem solving

- Decision-making

- Shared decision-making

- Patient management

- Staff management

- Financial management

- Continuity of care

- Unusual encounters

- 'Heart-sink' patients

- Uncertainty

- The 'good old days'

- Controversy/debate

- Life as a registrar

- Learning and teaching

- Not knowing what to do

- My first day in a practice

- Prioritising

- Handling complaints

- Handling stress

- Home visits

- Teamwork

- Work-life balance

You may submit as many entries as you wish (closing date for entries: 31 March 2009). Please email your submission(s) to Helen Farrelly at: hfarrelly@rcgp.org.uk

\section{David Orlans}

DOI: 10.3399/bjgp09X420176 